\title{
Effect of Transplanting Suprachiasmatic Nuclei from Donors of Different Ages into Completely SCN Lesioned Hamsters
}

\author{
Claire M. Kaufman and Michael Menaker \\ NSF Center for Biological Timing, Department of Biology \\ University of Virginia, Charlottesville, VA 22903, USA
}

\begin{abstract}
The suprachiasmatic nucleus (SCN) is the primary circadian pacemaker in mammals. Ralph and colleagues /14/ provided recent new evidence for this by transplanting SCNs between golden hamsters with different genetically determined periods and producing circadian rhythms of running wheel activity with periods characteristic of the donor. We have extended these studies in order to evaluate the age range of donor tissue that can be used for transplantation. SCN of hamsters from embryonic day 11 through postnatal day 12 can serve as functional grafts to restore rhythmicity to arrhythmic SCN lesioned animals. The time between SCN transplantation and onset of rhythmicity does not depend on the age of the donor. The presence of patches containing vasoactive intestinal peptide (VIP) immunoreactive cells is a good indicator of graft success, while its absence is correlated with a lack of transplant effect. The 18 day span during which SCN tissue can be harvested for transplantation should expand the uses to which this technique can be put. Our results also suggest that it would be advantageous to examine the age range of neural tissue that can be used in other transplantation models.
\end{abstract}

\section{KEY WORDS}

circadian rhythm, Syrian hamster, VIP

Reprint address:

Claire M. Kaufman

NIH/NIDDK-LN

Building 8, Room 111

Bethesda, MD 20892-0008, USA

\section{INTRODUCTION}

The technique of transplantation was successfully used by Ralph and colleagues in 1990 /14/ to show that the characteristics of the suprachiasmatic nucleus (SCN) itself determined circadian period. The protocol employed involved placing the SCN of a mutant fetal hamster with a circadian period of $20 \mathrm{~h}$ into a $24 \mathrm{~h}$ wild-type adult hamster which had been rendered arrhythmic by lesion of its SCN. In other experiments donor and host genotypes were reversed. SCN transplants which restored circadian rhythms of wheel running activity always did so with a period characteristic of the donor.

All previous attempts at SCN transplantation in rodents have utilized tissue harvested during late gestation or within a day of birth. It has been generally accepted that neuronal tissue should be used for transplantation when it is still undergoing neurogenesis or shortly thereafter. In the golden hamster gestation lasts 16 days and the suprachiasmatic nuclei themselves undergo neurogenesis from embryonic day (E) 9.5-13 /2,3/. In the rat gestation takes 22 days and $\mathrm{SCN}$ neurogenesis occurs between E 14-17/1/. The reported age range of hamster SCN tissue used for transplantation was from E 13 to $15 / 5,8 /$, and rat SCN tissue from E $17 / 6 /$ to postnatal day (P) 1 $/ 16 /$, where day 0 is the day of birth.

The research described here focuses on defining the age range of donor SCN tissue that yields successful transplants. We tested early fetal tissue to gain insight into the importance of graft maturation, and examined older postnatal tissue because working with larger brain structures allows more precise excision and greater ease of manipulation of donor SCN prior to implantation. In addition, we examined the characteristics of the restored rhythm, as well as the expression in the graft of two antigens 
normally abundant in the intact SCN but not in surrounding hypothalamic areas: glial fibrillary acidic protein (GFAP) /12/ which is found in astrocytes and vasoactive intestinal peptide (VIP) $/ 9,17 /$.

\section{METHODS}

All methods were as described previously $/ 14,19 /$, except where specifically noted. Briefly, adult male Lakeview Golden-Outbred hamsters (Mesocricetus auratus; Charles Rivers, Wilmington, MA) were anesthetized, positioned stereotaxically (+0.6 mm AP, $-8.3 \mathrm{~mm} \mathrm{~V}$ from bregma; nosebar $-2.0 \mathrm{~mm}$ ), and a platinum-iridium electrode was lowered through a hole drilled in the skull. The lesion was made with a $4 \mathrm{~mA}$ current passed for 15 sec. The electrode was removed, Gelfoam was placed over the hole, and the skin was closed with surgical staples.

Implantation did not immediately follow the lesion, but was performed 17 to 37 weeks later. Implants were prepared as previously described /14/. However, in this case, all donors were homozygous period mutants that had been bred in our colony and which would have displayed circadian periods of about $20 \mathrm{~h}$ if allowed to mature. All hosts were wild-type hamsters. In order to collect fetal SCN tissue, pregnant dams received a lethal dose of sodium pentobarbital. Fetuses were exposed by caesarian section and rapidly decapitated. Postnatal animals were decapitated. Heads were placed in medium [60\% Basal Eagle's Medium, with Earl's Salts and without L-glutamine; 40\% Hanks' Balanced Salt Solution (BME-HBSS)] at $36^{\circ} \mathrm{C}$ and further dissected to isolate a block of SCN-containing tissue (1-2 $\mu \mathrm{l})$.

For implantation of SCN tissue, host animals were once more anesthetized and placed in a stereotaxic apparatus (David Kopf, Tujunga, CA). The tissue was implanted through the preexisting hole by means of a glass cannula and plunger into the base of the third ventricle, the site of the SCN lesion. The wound was closed as after the lesion.

Locomotor activity was recorded from host animals individually housed in cages with running wheels, given unlimited access to food and water, and kept in constant darkness in light-tight boxes.
Activity recordings were accomplished as previously described /19/. Data were analyzed using the chi-squared periodogram /18/. The criteria used by Vogelbaum and Menaker /19/ were followed except that test periods ranges from 19 to $21 \mathrm{~h}$ (the period range of homozygous mutant hamsters /13/) in $6 \mathrm{~min}$ intervals, and rhythms were considered significant only if periodogram peaks (each peak having a confidence level of $p<0.05$ ) continued for more than 5 consecutive days. Latency to the onset of rhythmicity (i.e., the time from implantation to appearance of the donor rhythm) was determined by three independent observers who analyzed activity records of animals assigned coded identifications to eliminate bias. These values were then statistically analyzed using a one-way ANOVA followed by a post hoc Scheffe test. Only surviving animals having complete SCN lesions and grafts as determined histologically were included in these studies. For calculation of percentage survival after transplantation, only completely SCN lesioned animals (as determined histologically) were included; lesion success for those animals dying prior to histological analysis was determined on the basis of behavior $(9 / 75 ; 8$ of those 9 were transplanted with tissue from E 10, E 11, or P 28 donors).

Transplant histology was examined in all surviving animals. After locomotor activity data were collected, animals were anesthetized with a lethal dose of halothane by inhalation. They were perfused intracardially with physiological saline (0.9\%) containing $150 \mathrm{IU}$ heparin $/ 10 \mathrm{ml}$, followed by Zamboni's fixative ( $\mathrm{pH}$ 7.4) containing $4 \%$ formaldehyde in $0.1 \mathrm{M}$ phosphate buffered saline (PBS; $0.1 \mathrm{M}$ phosphate, $0.9 \%$ saline) with picric acid (15\%). After perfusion, brains were removed and postfixed at $4^{\circ} \mathrm{C}$. One day before sectioning, brains were cryoprotected by immersion in $25 \%$ sucrose in 0.01 M PBS. Brains were frozen and cut at a section width of $50 \mu \mathrm{m}$.

Free floating sections were washed in PBS-T (0.01 M phoshpate, $0.9 \%$ saline, $0.2 \%$ Triton X$100 ; \mathrm{pH} 7.4$ ) and incubated for $30 \mathrm{~min}$ in a $0.01 \mathrm{M}$ PBS solution containing $0.003 \%$ hydrogen peroxide followed by several washes in PBS. Sections were incubated for $1 \mathrm{~h}$ at room temperature in normal goat serum (1:30 PBS) and placed directly into anti-VIP (RIN 7161; Peninsula 
Laboratories, Belmont, CA) diluted 1:1000 or antiGFAP (ICN Bio-medicals, Costa Mesa, CA) diluted 1:500 in PBS-T for $72 \mathrm{~h}\left(24 \mathrm{~h} 22^{\circ} \mathrm{C}\right.$ : $48 \mathrm{~h}$ $4^{\circ} \mathrm{C}$ ). Sections were washed in PBS and sites of antibody:antigen binding were tagged by an avidinbiotin peroxidase procedure (Elite $\mathrm{ABC}$ Kit, Vector Labs, Burlington, CA). Following this, sections were washed in $0.05 \mathrm{M}$ Tris buffer ( $\mathrm{pH} 7.3$ ) before incubation with $0.0025 \%$ diaminobenzidine (DAB) containing $0.003 \%$ hydrogen peroxide for about 4 min. Sections mounted onto gelatin-coated slides were dehydrated through graded alcohols into xylene where they remained for a minimum of $4 \mathrm{~h}$. Sections were then rehydrated in distilled water and immersed in $0.2 \%$ osmium tetroxide $2 \mathrm{~min}$ to intensify staining. Histological analysis and photography were performed with a Zeiss Axiophot photomicrograph system.

\section{RESULTS}

As can be seen in Figure 1, donor SCN from $\mathrm{E} 11$ to $\mathrm{P} 12$ produced functional grafts in completely SCN lesioned hosts (Table 1 ). The age of the donor SCN producing the largest percentages of host animals with transplants that displayed
TABLE 1

\begin{tabular}{lcc}
\hline Age (days) & Mortality $^{\mathrm{a}}$ & Donor Rhythm $^{\mathrm{b}}$ \\
\hline E 10 & $4 / 5$ & $0 / 1$ \\
E 11 & $2 / 7$ & $2 / 5$ \\
E 12 & $1 / 5$ & $1 / 3$ \\
E 14 & $0 / 11$ & $9 / 10$ \\
P 2 & $0 / 6$ & $5 / 6$ \\
P 3 & $1 / 8$ & $4 / 5$ \\
P 4 & $0 / 8$ & $5 / 8$ \\
P 6 & $0 / 5$ & $4 / 5$ \\
P 8 & $0 / 5$ & $2 / 5$ \\
P 10 & $0 / 5$ & $3 / 5$ \\
P 12 & $0 / 6$ & $1 / 4$ \\
P 28 & $0 / 4$ & $0 / 1$ \\
\hline
\end{tabular}

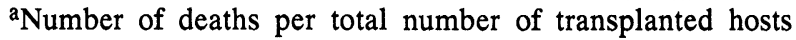
with complete SCN lesions

bumber of hosts displaying a donor rhythm per total number of hosts having a discernable transplant and complete SCN lesion

$\mathrm{E}=$ embryonic, $\mathrm{P}=$ postnatal

donor periods were E 14 and P 2. Even transplanted SCN from P 10 animals yielded clear circadian rhythms of wheel running activity with the approximately $20 \mathrm{~h}$ period of the donor (Fig. 2).

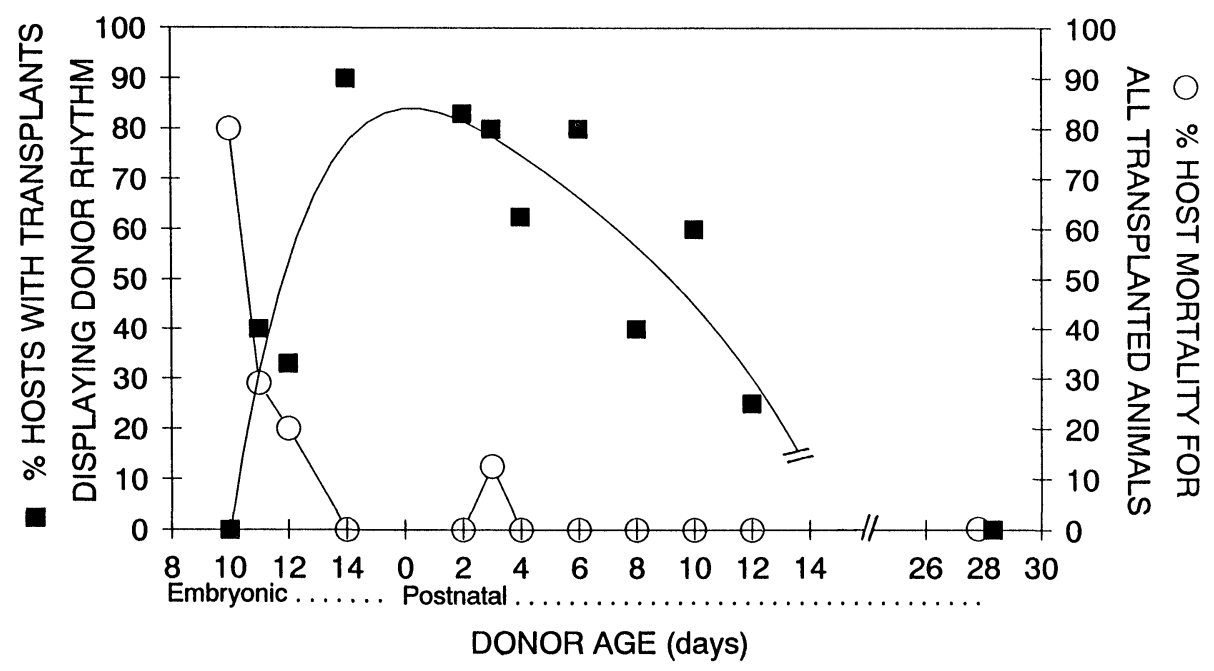

Fig. 1: Effect of $20 \mathrm{~h}$ mutant SCN transplanted into wild-type host hamsters with complete SCN lesions. E 11 to P 12 hamsters SCN produced functional transplants. Percentages of completely SCN lesioned hosts with histologically detected grafts having a period characteristic of the period mutant hamster varied with age of donor ( $\square$; regression line was calculted with a 5 th order polynomial equation). Mortality of all transplanted hosts was higher with the use of younger donors (O). 


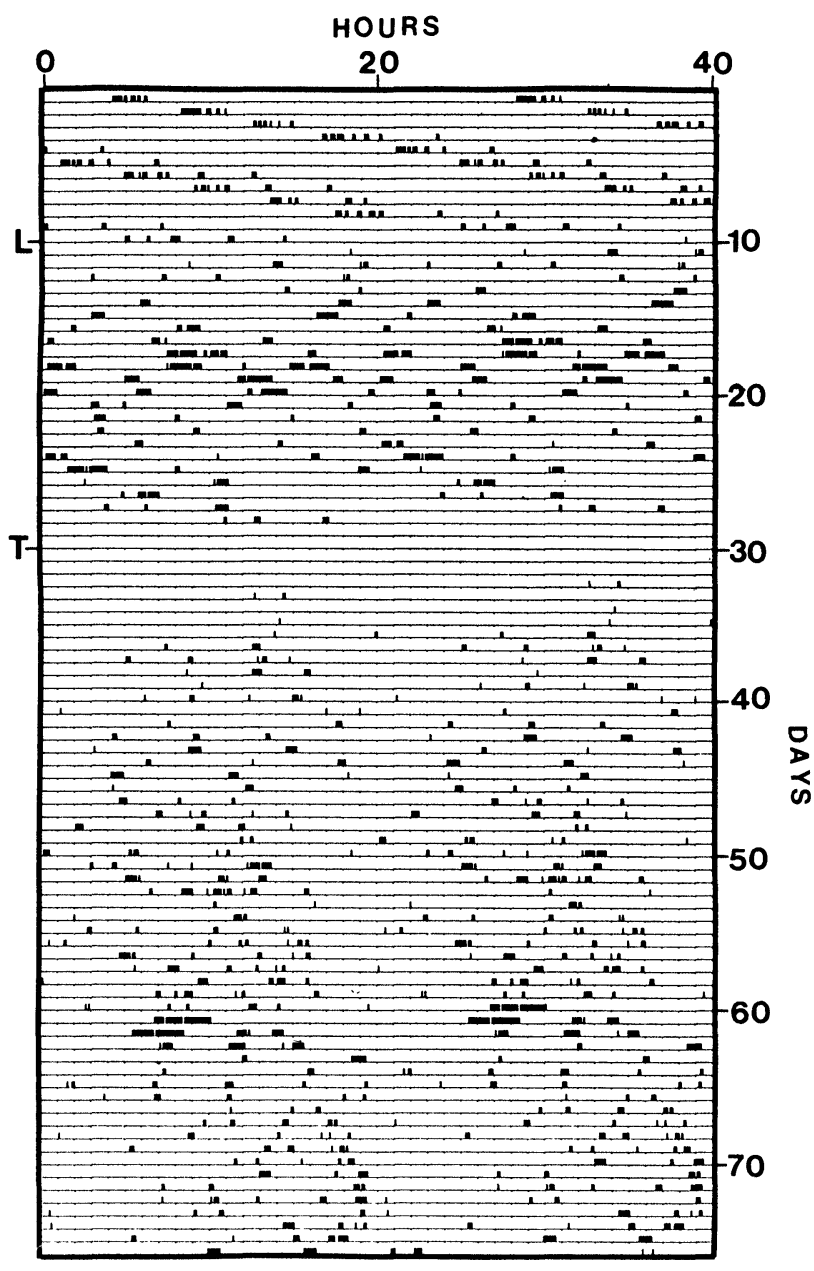

Fig. 2: Activity rhythm of a host before and after SCN lesion and transplantation with a P $10 \mathrm{SCN}$. The activity is doubled-plotted such that two consecutive $20 \mathrm{~h}$ days (the approximate length of the donor's period) are placed next to each other on one line and the latter day is repeated beneath the former. $\mathrm{L}=$ day of lesion. $\mathrm{T}=$ day of transplant.

Mortality occurring after the day of transplantation was highest when donors were from the youngest groups (Fig. 1 and Table 1). Tissue from E 10 fetuses was extremely difficult to isolate surgically both because the brain was in a primitive state and because the head was quite soft and small. As a result, the graft often contained cartilage from the skull as well as a larger area of the brain. In all cases $(\mathrm{N}=5)$ this led to illness or death. Histological analysis revealed volume increase of the graft.
Several grafts from animals older than $P 10$ appeared to have undergone degeneration within the host. As neonatal animals mature and particularly around the time the optic chiasm becomes myelinated ( $\mathrm{P} 10$ in the rat $/ 10 /$ ), the excised SCN (which inevitably contains part of the optic chiasm) increases in firmness. This may have contributed to the poor graft success seen when older donors were used. The characteristics of these more mature implants may result in physical isolation from nutrients, hormones, or necessary neural connections.

We compared the presence of patches of cells and fibers immunopositive for VIP with the presence of a circa-20 h rhythm characteristic of the donor SCN as determined either by periodogram analysis or by visual observation by three independent observers. Though there was some disagreement between the computational and visual methods of period analysis in terms of which transplants yielded donor activity rhythms, the percentage correlation of donor rhythm presence and of VIP patches within the graft was similar for the two period analysis methods. Patches of VIP immunopositive cells could be seen in all but three successful transplants as defined by periodogram analysis (N=36; Fig. 3 - immunocytochemistry; Fig. 4 - behavior). In one of the exceptions the transplant clearly conferred a $20 \mathrm{~h}$ rhythm, though no VIP stained cells could be detected. The remaining two grafts without apparent VIP also conferred an activity rhythm having a period consistent with the donor phenotype according to chi-squared periodogram analysis; however, the three observers did not find such a rhythm in the records. Of the 22 grafts in animals with complete SCN lesions that showed no statistically significant donor rhythm by periodogram analysis (e.g., Figs. 5 and 6), only three had patches of VIP immunoreactive cells and fibers. One of these appeared (visually) to be totally arrhythmic. Although chisquared periodogram analysis also failed to detect a significant circa-20 h rhythm for the other two transplanted animals, visual inspection of the locomotor activity revealed apparently clear donor rhythms. If one were to use only visual observations of activity records and compare the results with the assumption that grafts having patches of VIP stained cells were indicative of successful 

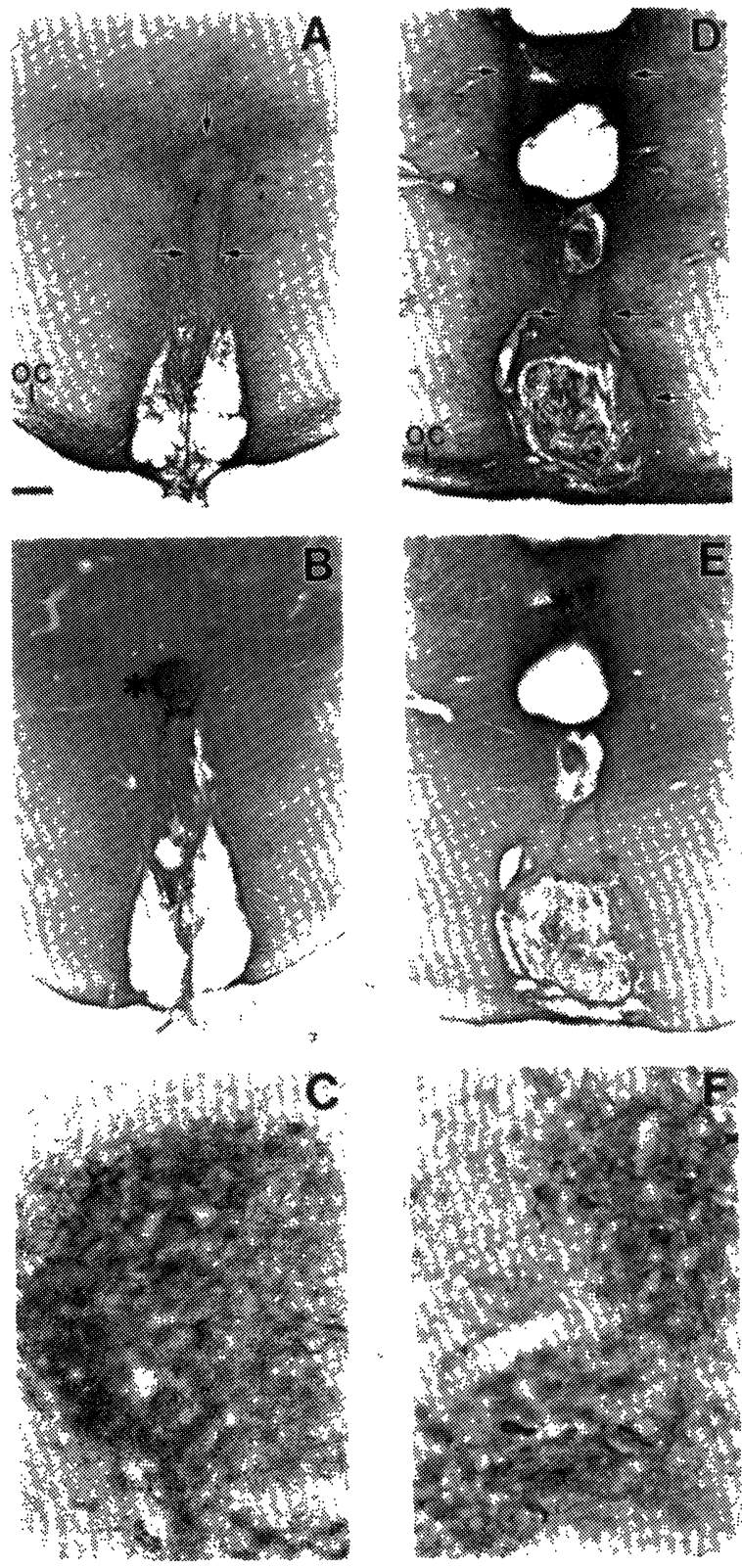

Fig. 3: Photomicrographs showing GFAP and VIP stained alternate sections from successful SCN grafts as determined by periodogram analysis. Two different grafts of P 4 SCN (A-C and D-F) are represented. Corresponding behavioral data is shown in Fig. 4A and $\mathbf{B}$, respectively. $\mathbf{A}$ and $\mathbf{D}$ : GFAP staining near graft borders which are indicated by arrows. "OC" indicates the optic chiasm. $\mathbf{B}$ and $\mathbf{E}$ : Patches of VIP immunoreactive cells and fibers typical of functional transplants. * marks the location of VIP immunoreactive patches in $\mathbf{B}$ and $\mathbf{E}$. These patches are seen at an $8 \mathbf{x}$ higher magnification in $\mathbf{C}$ and $\mathbf{F}$, respectively. Scale bar $=200 \mu \mathrm{m}$ for $\mathbf{A}, \mathbf{B}, \mathbf{D}, \mathbf{E} ; 25$ $\mu \mathrm{m}$ for $\mathbf{C}$ and $\mathbf{F}$. transplants, there would be disagreement in only two out of 58 cases - one animal with no VIP patch but having a visually evident rhythm and one with a VIP patch but no visually evident rhythm. There was a $97 \%$ correlation between the presence of VIP patches and of rhythmicity characteristic of the donor as determined by visual inspection, and this correlation is a little higher than that between the presence of VIP patches and of a donor rhythm as determined by chi-squared periodogram analysis (90\%). Though the three possible criteria for transplant success (i.e., the presence of a patch of VIP positive cells, visual analysis of running wheel activity, and chi-squared periodogram analysis) did not agree in every case, the percentage of functional grafts identified by each criterion was similar. While it is difficult to select one method of determining transplantation success as being superior, it may be that combining immunocytochemical analysis of the graft with a method of period analysis would provide the best means of assaying transplant success.

We noted several transplanted animals that appeared to have complete SCN lesions as judged histologically, but in which $24 \mathrm{~h}$ host activity rhythms were still detectable. Four such animals had activity rhythms characteristic of only the host with no evidence of donor rhythm, while one displayed rhythms characteristic of both the host and donor. Using a protocol similar to the one described in this paper, Vogelbaum and Menaker (manuscript in preparation) have found that if a host rhythm is detectable after transplantation, it is less likely that a donor rhythm will appear.

GFAP was detectable in all grafts, both successful (Fig. 3A and C) and unsuccessful (Fig. 5A). Levels of GFAP seemed to have no correspondence to restoration of rhythmicity, but were difficult to quantify. The GFAP staining induced after trauma resulting from implantation, however, made the graft borders more easily definable.

Latency to the onset of rhythmicity was measured from the time of implantation and scored by three independent observers and by the chisquared periodogram. Mean latency for all ages determined visually was 20.2 days and determined by periodogram was 17.1 days. Latency showed no correlation to donor age ( $p=0.1$, Fig. 7$)$. 


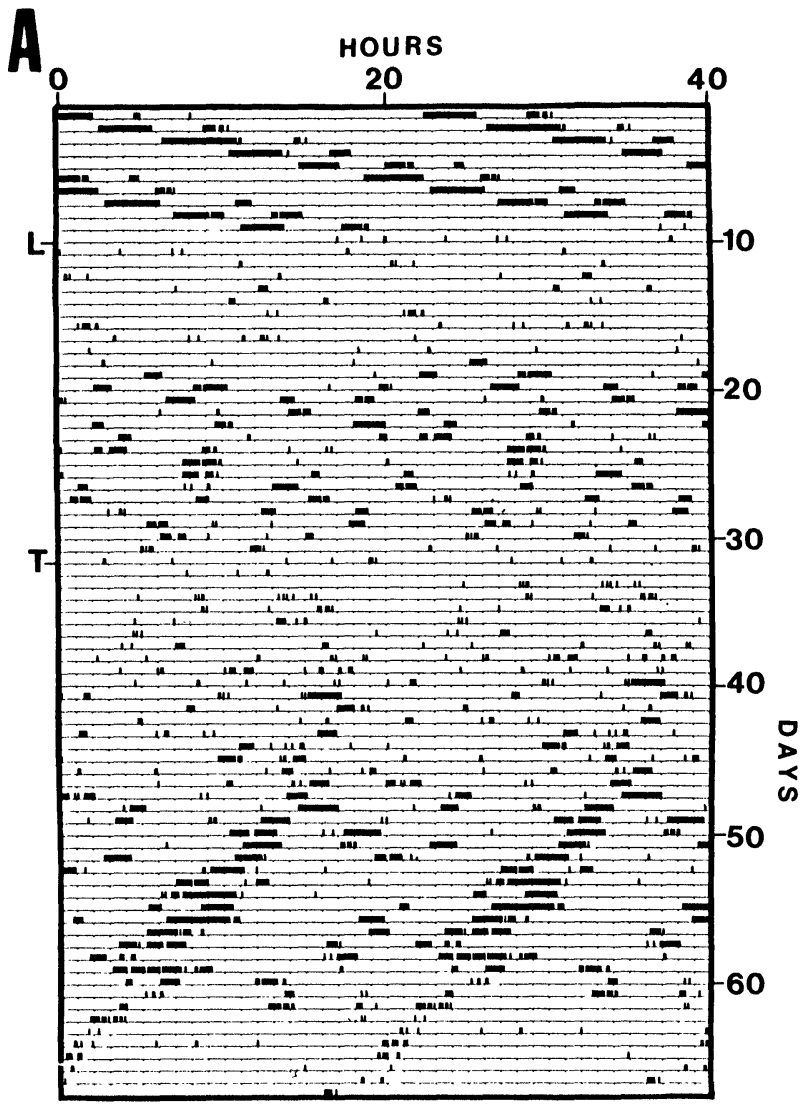

Fig. 4: Activity rhythms from the successful transplants depicted in Fig. 3. A and B: Locomotor behavior in two animals that received $\mathrm{P} 4 \mathrm{SCN}$ grafts (Fig. 3A$\mathrm{C}$ and 3D-F, respectively) double-plotted as previously described with a day-length of $20 \mathrm{~h} . \mathrm{L}=$ day of lesion. $\mathrm{T}=$ day of transplant.

\section{DISCUSSION}

We were able to transplant successfully the SCN of period mutant $(20 \mathrm{~h})$ hamsters, aged from embryonic day 11 through postnatal day 12 , into the third ventricle of wild-type SCN lesioned hosts. This 18 day span begins during SCN neurogenesis and lasts approximately until the number of synapses per unit area reaches adult levels (P 10 for the rat $/ 10 /)$. Since it was possible to transplant tissue that was in the midst of active neurogenesis, maturation of the transplanted SCN may have continued within the host. Based on the previous findings of Ralph and colleagues /14/, we have no reason to believe the results would be different if the host and donor genotypes were reversed.

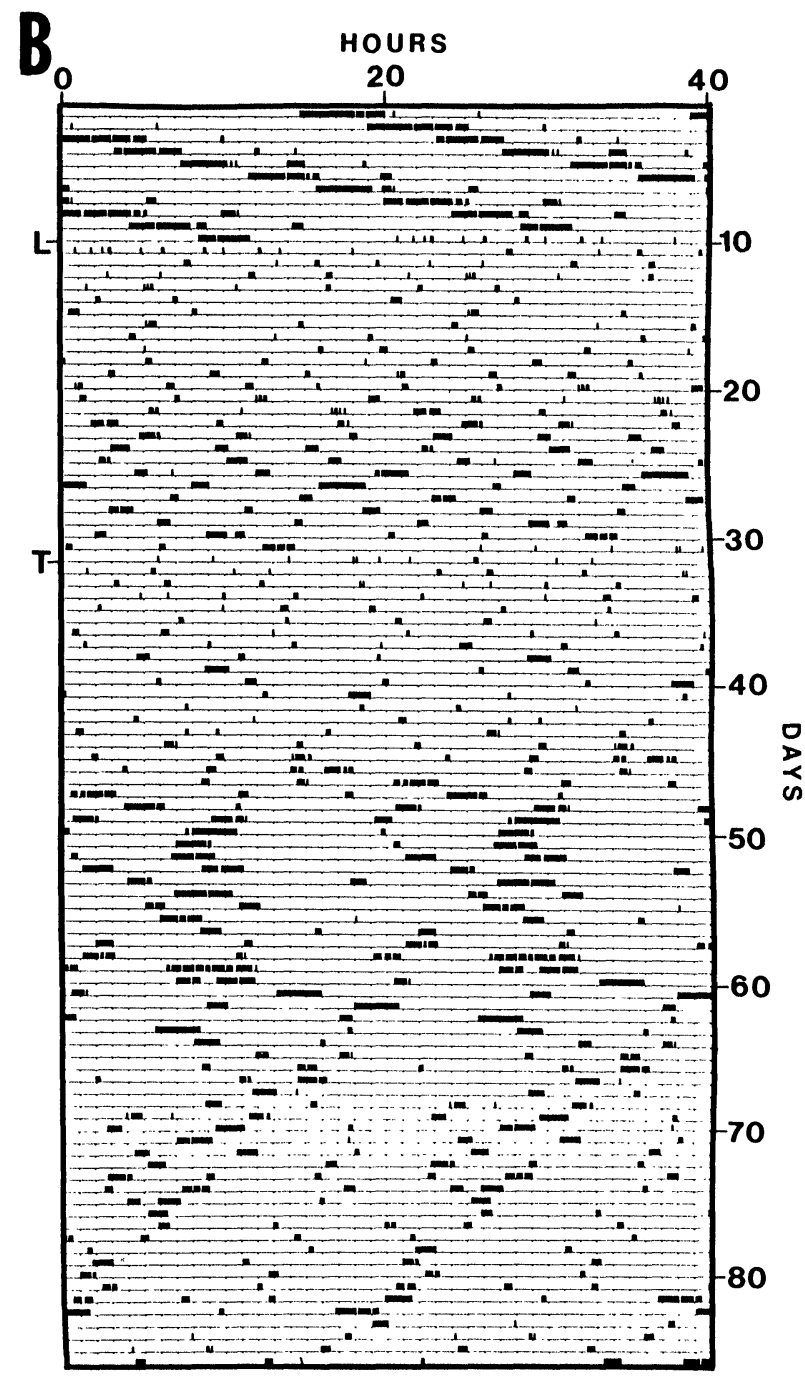

The age of donor tissue had no relationship to the latency to rhythm onset after transplantation. Romero and Silver /15/ have suggested that latency to rhythm onset may be due to the time required to renew peptidergic expression after a developmental delay resulting from transplantation. Our findings suggest this is not the case; if it were, older SCN tissue in which levels and patterns of neuropeptides resemble those of the adult $/ 4,7,11 /$ should produce rhythmicity with a shorter latency than younger tissue. On the contrary, younger fetal SCN tissue does not appear to require more time than older postnatal grafts to develop or to make appropriate connections with the host. The possibility exists, however, that a small difference in latency related to donor age is obscured because of disruption of 

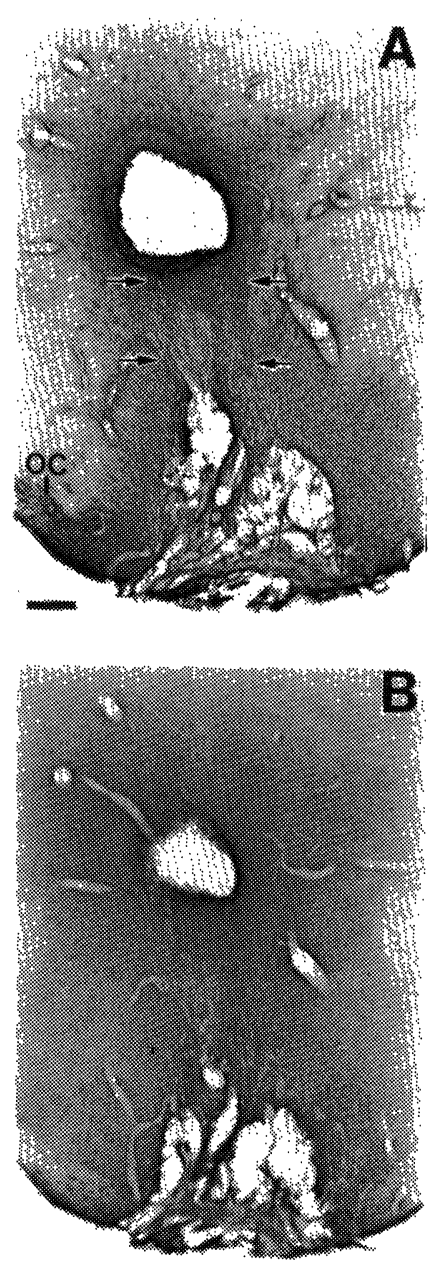

Fig. 5: Photomicrograph of an unsuccessful graft as determined by chi-squared periodogram analysis. A: GFAP staining of an unsuccessful $P 10$ graft and its borders. Arrows point to position of graft borders. "OC" indicates the optic chiasm. B: The alternate section showing a lack of VIP staining in the graft. Scale bar $=200 \mu \mathrm{m}$. Locomotor activity from this animal is seen in Fig. 6.

running wheel activity caused by trauma induced by the transplantation process.

The levels of VIP staining did not appear to correspond to either age of the donor SCN or transplant success. Nevertheless, there was a high correlation between transplant success and presence of at least one patch of VIP immunoreactive cells and fibers which resemble intact SCN. These findings suggest that VIP may be necessary for

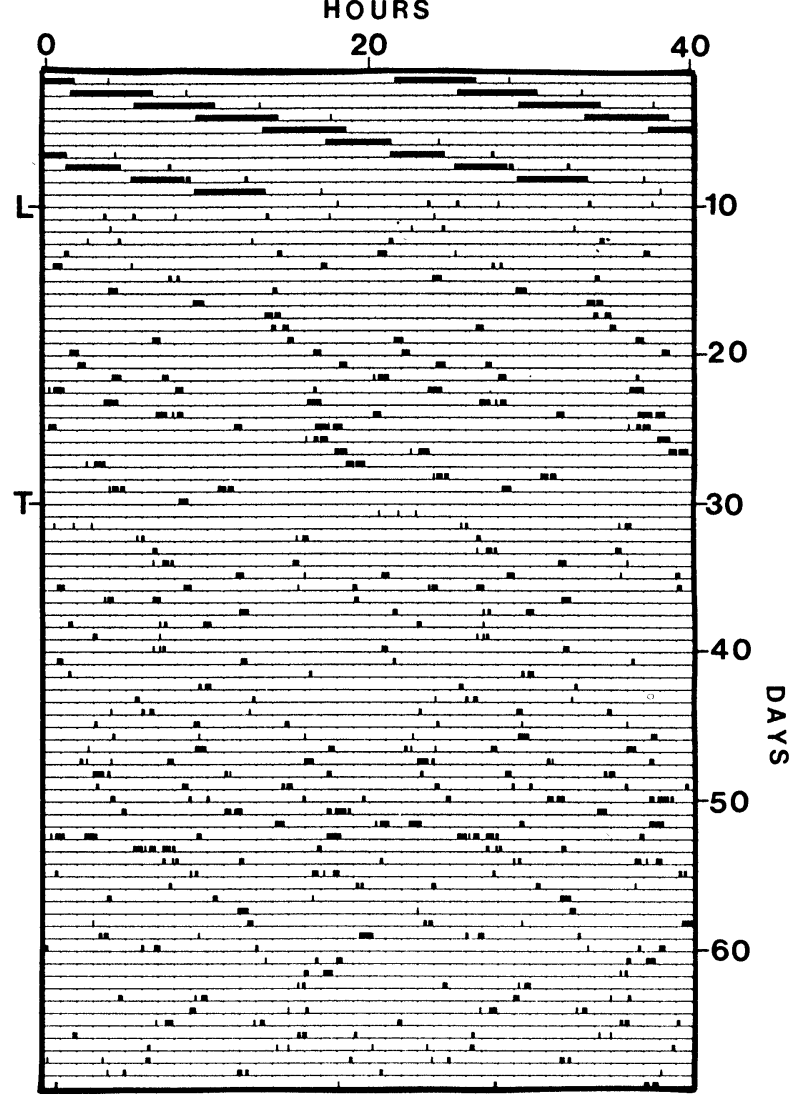

Fig. 6: Absence of donor phenotype as determined by chisquared periodogram analysis. Activity of an animal bearing the $P 10$ transplant pictured in Fig. 5 , double-plotted with a day length of $20 \mathrm{~h}$ as in previous figures. $\mathrm{L}=$ day of lesion. $\mathrm{T}=$ day of transplant.

transplant function. This type of VIP staining appears to be as sensitive an assay of transplant success as chi-squared periodogram analysis of behavior. GFAP staining aided in the localization of graft borders.

Our findings extend the age range over which donor SCN tissue from hamsters can be expected to confer rhythmicity successfully. The extended range adds flexibility to breeding and surgery schedules. Because of their larger size, SCN from older postnatal animals can be excised more precisely than can SCN from fetuses or young neonates. Better results should be attainable by labeling 


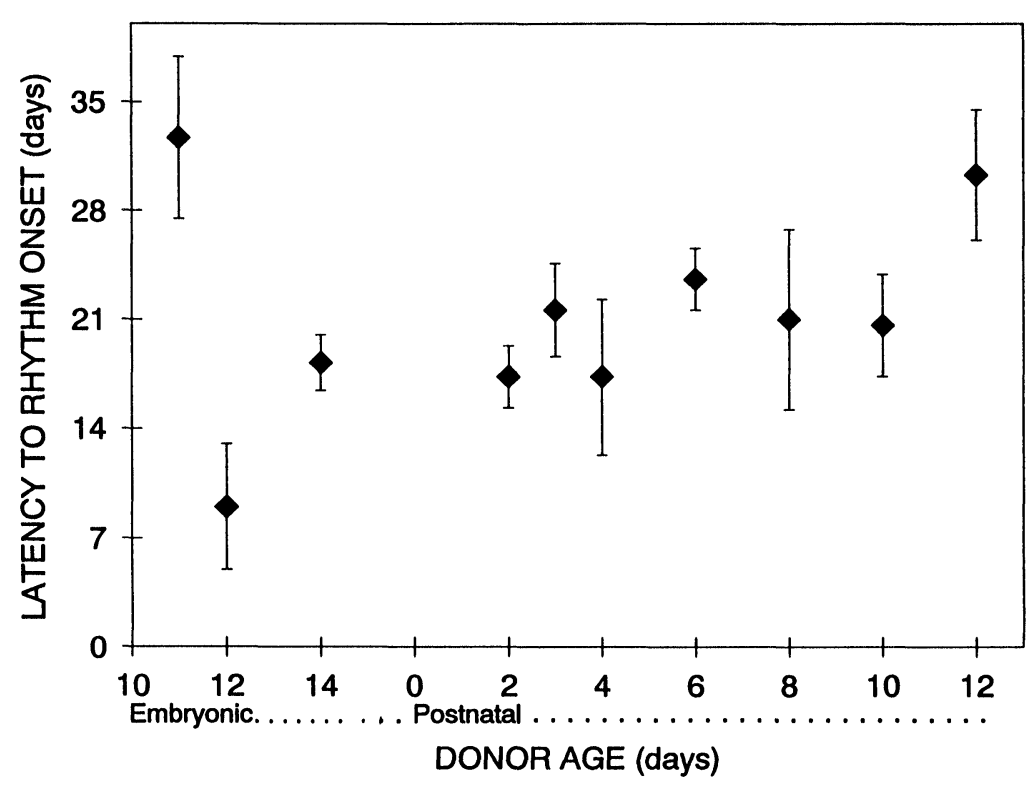

Fig. 7: Latency to onset of rhythm conferred by SCN transplants. There was no significant correlation ( $\mathrm{p} \leq 0.1$ ) between donor age and the number of days from transplantation to donor activity rhythm appearance. Symbols represent mean \pm SEM for values estimated by 3 independent observers.

postnatal rather than embryonic transplant tissue in order to trace transplant efferent fibers. Since mothers need not be killed if neonates are used as donors, results from transplantations using successive litters from the same dam can be compared. Additionally, the light:dark cycle could be used over a matter of weeks to impart an identifiable characteristic to the rhythm other than period (e.g., phase of activity) which might then be transplantable.

The 18 day span during which donor SCN may be harvested from hamsters for successful transplantation suggests that the SCN may be capable of undergoing changes and adaptations during a lengthy maturation. This may be important for normal calibration of the developing clock by maternal cycles (daily rhythms of maternal hormones such as melatonin) and environmental cycles (e.g., light:dark).

Note added in proof: Romero et al. (Dev Brain Res 1993; 71: 45-52) have shown that SCN from P 3 hamsters can restore rhythmicity to SCN lesioned hosts.

\section{ACKNOWLEDGEMENTS}

The research reported was supported by Air Force Office of Scientific Research grant 90-0098 to M.M. C.M.K. Was also supported by the NSF Center for Biological Timing.

\section{REFERENCES}

1. Altman J, Bayer SA. The development of the rat hypothalamus. Adv Anat Embryol Cell Biol 1978; 100: 1-173.

2. Crossland WJ, Uchwat CJ. Neurogenesis in the central visual pathways of the golden hamster. Dev Brain Res 1982; 5: 99-103.

3. Davis FC, Boada R, LeDeaux J. Neurogenesis of the hamster suprachiasmatic nucleus. Brain Res 1990; 519: 192-199.

4. De Vries GJ, Buijs RM, Swaab DF. Ontogeny of the vasopressinergic neurons of the suprachiasmatic nucleus and their extrahypothalamic projections in the rat brain - presence of a sex difference in the lateral septum. Brain Res 1981; 218: 67-78.

5. DeCoursey PJ, Buggy J. Restoration of circadian locomotor activity in arrhythmic hamsters by fetal SCN transplants. Comp Endocrinol 1988; 7: 49-54. 
6. Drucker-Colín R, Aguilar-Roblero R, GarcíaHernández F, Fernández-Canino F, Rattoni FB. Fetal suprachiasmatic nucleus transplants: diurnal rhythm recovery of lesioned rats. Brain Res 1984; 311: 353357.

7. Ishikawa K, Frohman LA. Ontogenesis of peptidehistidine-isolucine (PHI)-containing neurons in the suprachiasmatic nucleus ( $\mathrm{SCN}$ ) of the rat. Brain Res 1987; 407: 144-148.

8. Lehman MN, Silver R, Gladstone WR, Kahn RM, Gibson M, Bittman EL. Circadian rhythmicity restored by neuronal transplant. Immunocytochemical characterization of the graft and its integration with the host brain. J Neurosci 1987; 7: 1626-1638.

9. Loren I, Emson PC, Fahrenkrug J, Björklund A, Altumets J, Hakanson R, Sundler F. Distribution of vasoactive intestinal polypeptide in the rat and mouse brain. Neuroscience 1979; 4: 1953-1976.

10. Moore RY, Bernstein ME. Synaptogenesis in the rat suprachiasmatic nucleus demonstrated by electron microscopy and synapsin I immunoreactivity. J Neurosci 1989; 9: 2151-2162.

11. Moore RY, Shibata S, Bernstein M. Developmental anatomy of the circadian system. In: Reppert SM, ed, Development of Circadian Rhythmicity and Photoperiodism in Mammals. Ithaca: Perinatology Press, 1989; 1-24.

12. Morin LP, Johnson RF, Moore RY. Two brain nuclei controlling circadian rhythms are identified by GFAP immunoreactivity in hamsters and rats. Neruosci Lett 1989; 99: 55-60.

13. Ralph MR, Menaker M. A mutation of the circadian system in golden hamsters. Science 1988; 241: 4225 4227.

14. Ralph MR, Foster RG, Davis FC, Menaker M. Transplanted suprachiasmatic nucleus determines circadian period. Science 1990; 247: 975-978.

15. Romero M, Silver R. Time course of peptidergic expression in fetal suprachiasmatic nucleus transplanted into adult hamsters. Dev Brain Res 1990; 57: 1-6.

16. Sawaki Y, Nihonmatsu I, Kawamura $H$. Transplantation of the neonatal suprachiasmatic nucleus into rats with complete bilateral suprachaismatic lesions. Neurosci Res 1984; 1: 67-72.

17. Sims KB, Hoffman DL, Said SI, Zimmerman EA. Vasoactive intestinal polypeptide (VIP) in mouse and rat brain: an immunocytochemical study. Brain Res 1980; 186: 165-183.

18. Sokolove PG, Bushell WN. The chi-square periodogram: its utility for analysis of circadian rhythms. J Theor Biol 1978; 72: 131-160.

19. Vogelbaum MA, Menaker M. Temporal chimeras produced by hypothalamic transplants. J Neurosci 1992; 12: 3619-3627. 

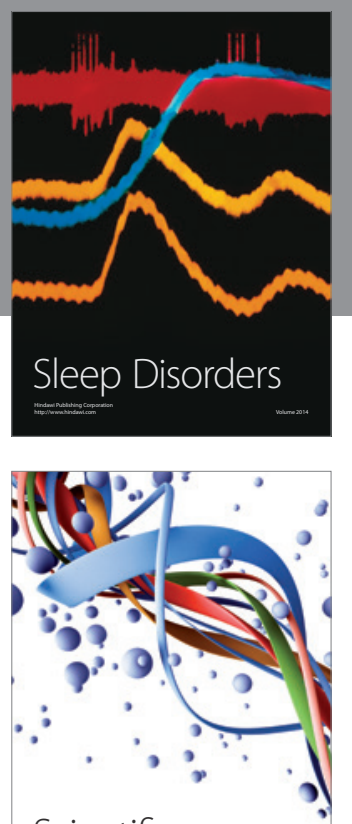

Scientifica
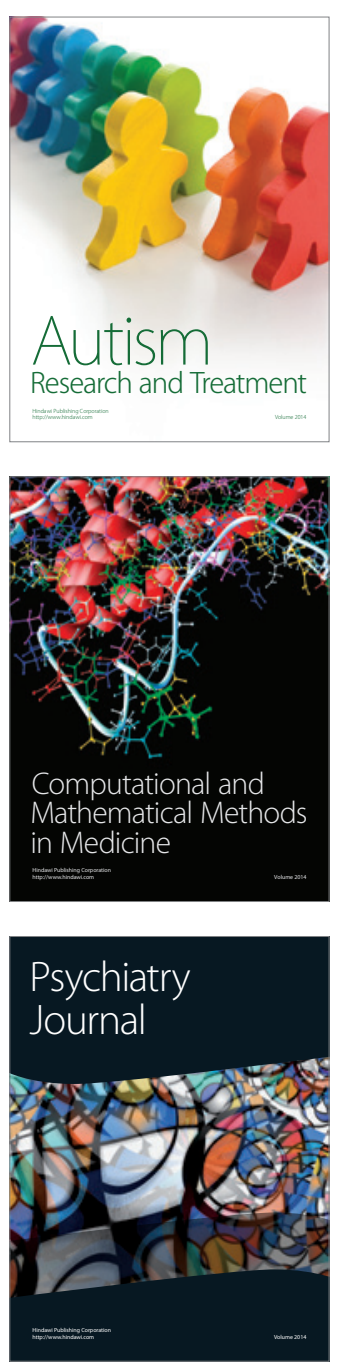
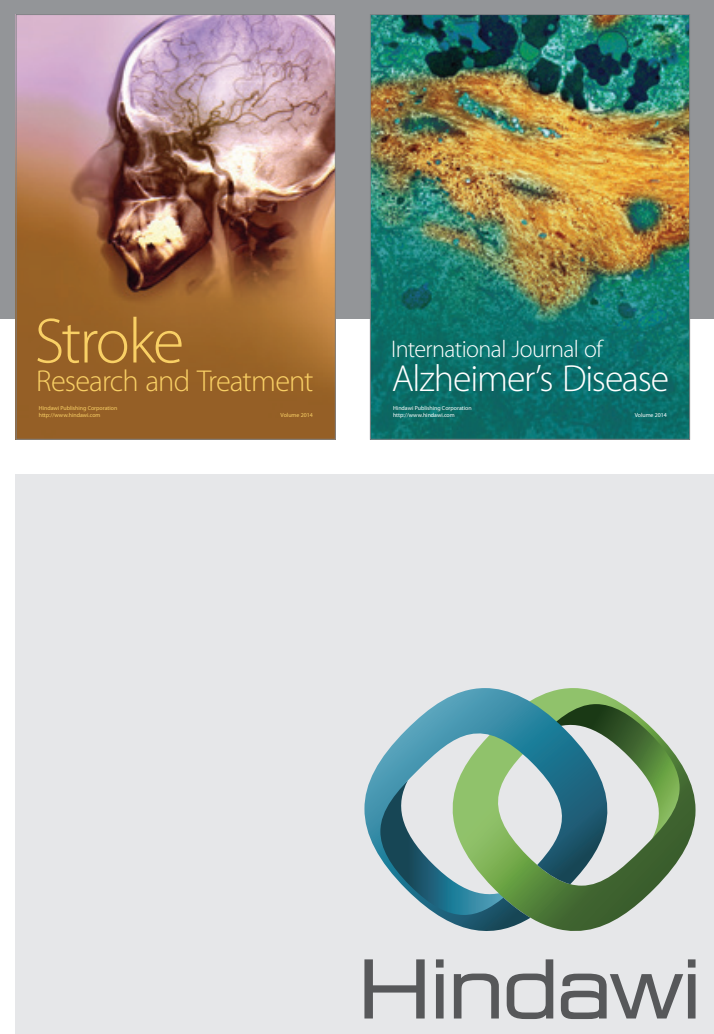

Submit your manuscripts at

http://www.hindawi.com
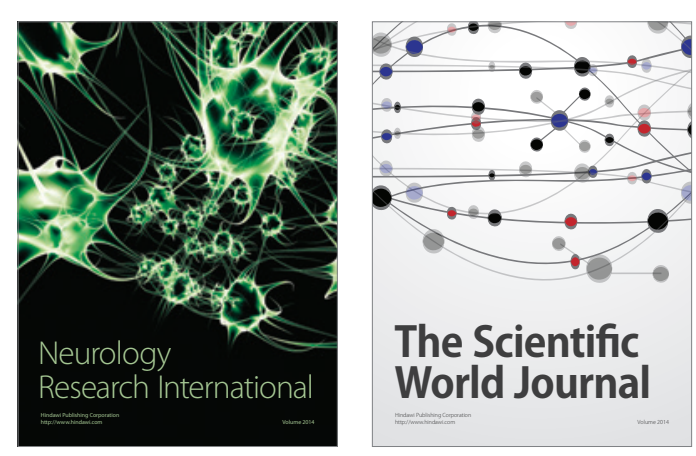

The Scientific World Journal

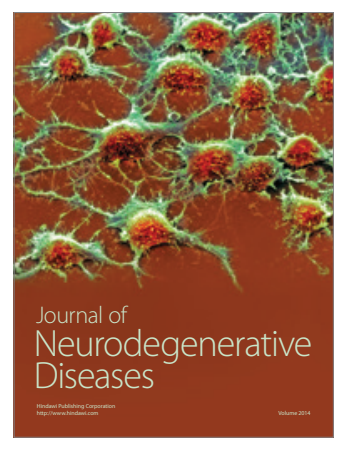

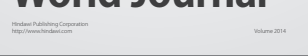

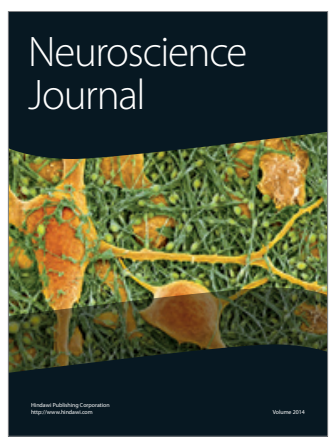

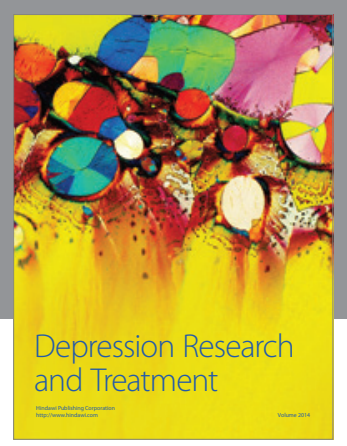
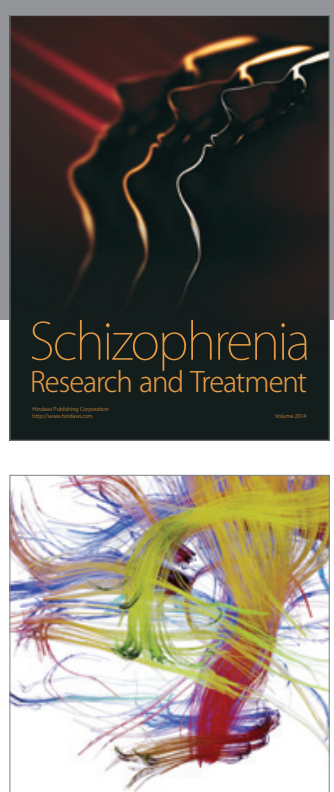

Brain Science

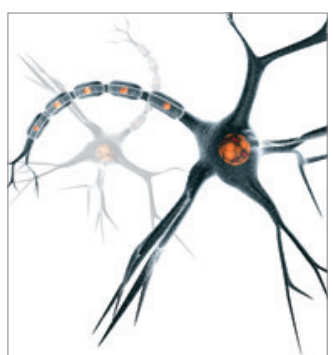

Neural Plasticity
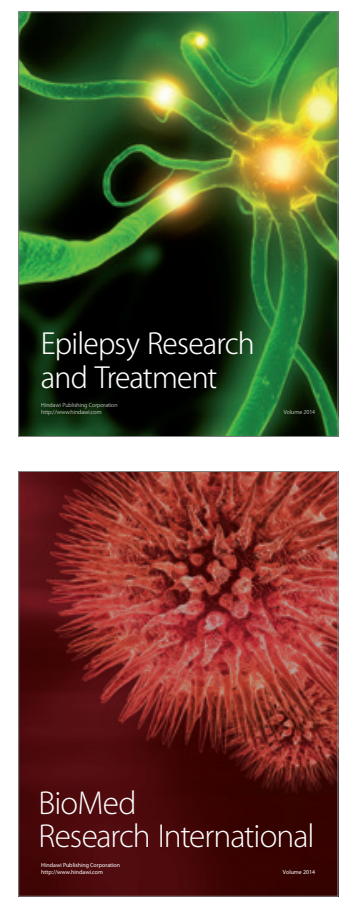

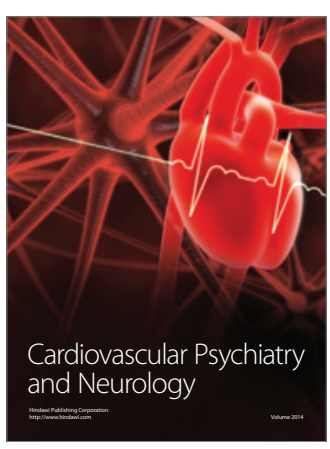

Parkinson's

Disease
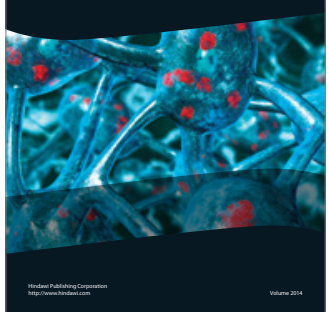